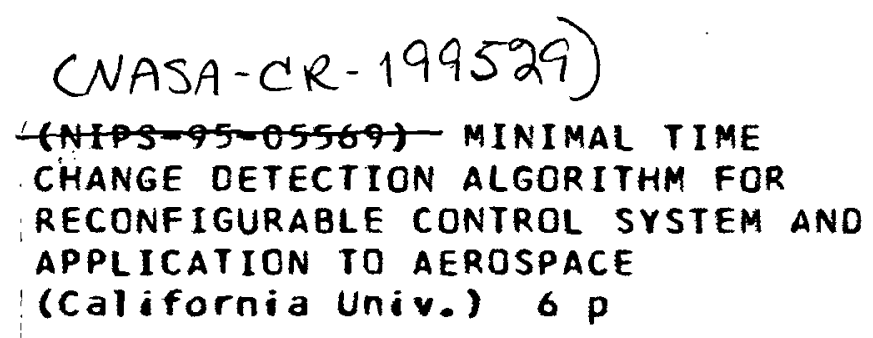

N96-13234

Unclas

$63 / 18 \quad 0073241$ 


\title{
MINIMAL TIME CHANGE DETECTION ALGORITHM FOR RECONFIGURABLE CONTROL SYSTEM AND APPLICATION TO AEROSPACE*
} 5569

\author{
SUNGWAN KIM \\ Electrical Engineering Department \\ School of Engineering and Applied Science \\ University of California, Los Angeles
}

\begin{abstract}
System parameters should be tracked on-line to build a reconfigurable control systems even though there exists an abrupt change. For this purpose, a new performance index that we are studying is the speed of adaptation - how quickly does the system determine that a change has occurred? In this paper, a new, robust algorithm that is optimized to minimize the time delay in detecting a change for fixed false alarm probability is proposed. Simulation results for the aircraft lateral motion with a known or unknown change in control gain matrices, in the presence of doublet input, indicate that the algorithm works fairly well. One of its distinguishing properties is that detection delay of this algorithm is superior to that of Whiteness Test.
\end{abstract}

Key Words. Change Detection, Intelligent Control, Fault Tolerant System, Likelihood Ratio, False Alarm

\section{INTRODUCTION}

Designing a reconfigurable control system for an aerospace vehicle which operates throughout a wide range of flight environmental conditions that affect its dynamic characteristics is very attractive. The problem of identifying aircraft system parameters from flight test data and their application to flight control has been successfully solved for linear models, by Balakrishnan (1972), Iliff (1973), and Maine (1986), when there are no severe changes in aircraft and environment. Even when the control design incorporates a degree of robustness, system parameters may drift enough to degrade its performance below an acceptable level. In other words, we still need to develop a theory for a better control system when aircraft flies at a ligh angle of attack where the linear model is no longer valid and when the robust controller does not work due to large variations in system parameters or noise levels.

Adaptive controls are considered to be a promising approach to give us a possible solution. An

*This work was supported by NASA Ames Research Center, DFRF through UCLA Flight Systems Research Center(FSRC), Grant Number NCC 2-374. adaptive control problem arises whenever system parameters are unknown or are subject to unknown variation which is usually a small change. It is also of increasing practical importance, since adaptive techniques are being used more and more in industrial control systems. However, this field is not mature yet, and only a set of scattered simulational results exists with no single quantitative measure for judging performances. Taking into account abrupt (or drastic) changes, which include any faults in the system, in statistical models appears as a natural complement of most of the adaptive techniques which track only slow variations of parameters. So, the detection of abrupt change is essential in the design of a reconfigurable control system for these types of adaptive problems. Therefore, the problem of detection, estimation, and diagnosis of changes in dynamical properties of signals or systems are considered, with particular emphasis on statistical methods for detection, to provide a general framework for change detection in signals and systems.

The development of the change detection problem was stimulated by Wald (1947) when 'Sequential Analysis' was published and a Sequential Probability Ratio Test was introduced. A 'sequen- 
tial process inspection scheme' was proposed by Page (1954) to detect a change in the mean by testing a weighted sum of the last few observations, i.e. a moving average. Named the 'PageHinkley' rule, Page pointed out that this rule is equivalent to performing a SPRT. The theoretical properties of this rule have been investigated for a long time from on-line and off-line points of view. The most significant works in that direction are Shiryayev (1978) and Lorden (1971). They formulated an 'Optimal Stopping' problem and proposed simple rules which are optimal in an appropriate sense. And the general problem of optimality of random processes in discrete time was considered and developed by Chow, Robbins, and Siegmund (1971). The problem of change detection has received growing attention during the last 20 years, as can be seen from survey papers and books (Basseville, 1980; Basseville and Benvensite, 1988; Basseville and Nikiforov, 1993; Wilsky, 1976). But, the development of change detection method is still a relatively new subject.

For reconfigurable control system, a new performance index that we are studying is the speed of adaptation - how quickly does the system determine that a change has occurred? A new, robust algorithm developed in this paper is called Minimal Time - Change Detection Algorithm (MTCDA) which minimizes the time delay in detecting a change for fixed false alarm probability.

A block diagram shown in Fig. 1 explains the reconfigurable control system for the aircraft control that is equipped with a CDA. Before the CDA detects a change, the regulator is designed based on the initial parameter values. But after the change is detected at time $\tau$, the CDA block generates an Activation Signal to re-initiate system identification. The system identification process finds a new set of parameter values proper for the changed system. The regulator uses the new parameter values to build a new control law. This process is continued until the new parameter set converges.

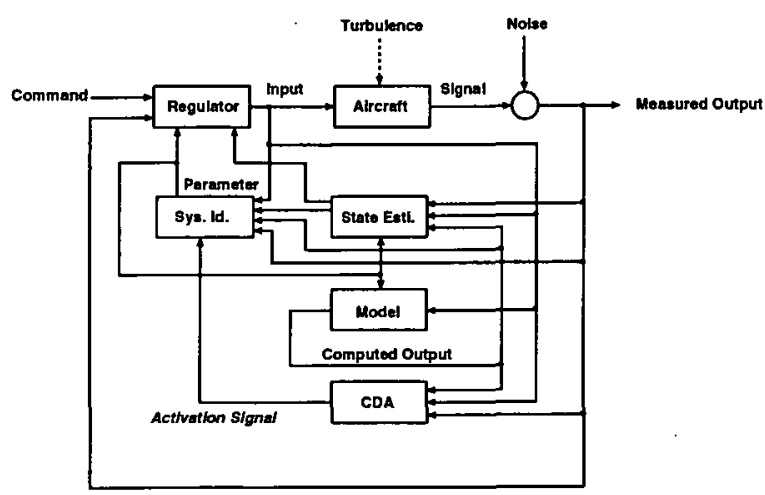

Fig. 1. A Block Diagram of Control System for Aircrafts with CDA
Algorithm complexity is definitely an issue for detection of each possible change and time of change. In spite of this complexity, we can have a control system which can detect its change and adapt it as quickly as possible even though the large amount of change is occurred at random time. Therefore, this MT-CDA will present a proper starting point for a reconfigurable control system in the theoretical point of view.

\section{MINIMAL TIME CHANGE DETECTION ALGORITHM}

\subsection{MT-CDA: Detection of a Known Change}

The following state space model is considered.

$x_{n+1}=A x_{n}+\left(B_{1}+\left(B_{2}-B_{1}\right) \chi(n-\theta)\right) u_{n}$

$v_{n}=C x_{n}+\left(D_{1}+\left(D_{2}-D_{1}\right) \chi(n-\theta)\right) u_{n}+G N_{n}$

where $\chi(m)=1$ if $m \geq 0$ and 0 otherwise. Based on this model, the basic problem to be solved is to detect a known change in control gain matrices $(B$ and $D)$ as quickly as possible in order to build a reconfigurable control system whose parameters could be switched from a known system to another known system after change detection. This is appropriate for a system which operates in two alternate driving points, and restricted to the class of models with a change of parameters which is known. The probability of making a false detection also needs minimized since the signals are disturbed by a white Gaussian noise.

We want to formulate an algorithm which finds the detection $\operatorname{time}(\tau)$ to minimize the average time delay

$$
E\left[(\tau-\theta)^{+}\right]
$$

subject to a given false alarm probability, i.e.

$$
\operatorname{Pr} .[\tau<\theta] \leq \alpha
$$

where $\alpha$ is given. 'Likelihood Ratio (change vs. no change)', which we shall define to be the ratio

$$
\frac{\text { Joint density of } v_{1}, \cdots, v_{n} \text { (with change) }}{\text { Joint density of } v_{1}, \cdots, v_{n} \text { (without change) }}
$$

is introduced and $p(\cdot), p_{\infty}(\cdot)$ denote the likelihood probability density function with and without changes at $\theta=k$. Then we define the following functional

$$
L_{n, k}=2 \log \frac{p\left(v_{1}, v_{2}, \cdots, v_{n} \mid \theta=k\right)}{p_{\infty}\left(v_{1}, v_{2}, \cdots, v_{n}\right)}
$$

and for each $n$,

$$
\ell_{n}=\max _{k \leq n} L_{n, k} .
$$


Now a decision rule for CDA is

$$
\tau=\min \left\{n: \ell_{n}>h\right\}
$$

where $\tau$ is a change detection time and $h$ is a threshold value computed from the probability of false alarm equation (Eq.(2)).

This algorithm stops the first time when $\ell_{n}$ exceeds a given threshold. This is based on Eq.(3)(5), so it can be regarded as 'Maximum Likelihood' treatment of the unknown change time, i.e. stop at $\tau=n$ when for some $k$ the observations $v_{k}, \cdots, v_{n}$ are significant.

By the contributions of Shiryayev (1978), Lorden (1971), and Kim (1993), there exists a change detection time and this procedure could be said optimal (at least asymptotically optimal), in the sense that it minimizes the detection delay for a fixed false alarm probability.

\subsection{M'T-CDA: Detection of an Unknown Change}

In this section, minimal time detection of an unknown change instead of a known change is considered, and its algorithm is derived. Multiple detectors are required to detect an unknown change, where the each detector is based on the known amount of change, in contrast to single detector required for the detection algorithm of a known change. The algorithm for the detection of only change time, which is a little bit simpler problem, is first derived and the detection algorithm of both time and amount of change is followed.

Detection of the Time of Change. The detection of change time is a significant problem in some applications, for example,

a. Fault detection: all the processes are stopped when a failure occurs and are re-started after the failure is fixed.

b. When a system requires the exact parameter set after a change, it is preferable to re-initiate the system identification just after detecting a change.

In adaptive system sense, the 2nd case is a popular one and a promising problem to be considered. To detect an unknown change, the CDA Block in Fig. 1 contains multiple change detectors. Each of change detection algorithm is based on a fixed known change, so each of detectors is basically the same one as derived in Section 2.1. By examining the Activation Signal for each $n$, the real Activation Signal at time $n$ is generated by the earliest one. The idea of this algorithm is that if there exists an unknown change, the exact (or the closest) detector will make Activation Signal at the earliest time. So the time of an unknown change will be detected with this algorithm if the amount of an unknown change is in the range of the multiple detector hypotheses.

To formulate this MT-CDA for an unknown change, we consider the following system model

$$
\begin{aligned}
& x_{n+1}=A x_{n}+\left(B_{1}+\left(B_{2}-B_{1}\right) \chi(n-\theta)\right) u_{n} \\
& v_{n}=C x_{n}+\left(D_{1}+\left(D_{2}-D_{1}\right) \chi(n-\theta)\right) u_{n}+G N_{n}
\end{aligned}
$$

where $B_{2}$, and $D_{2}$ are unknown. The only difference, compared to the Section 2.1, is that control gain matrices are unknown which stands for the unknown change. Like Section 2.1, we define the following functional, which is related to 'Likelihood Ratio', for each detector

$L_{n, k}^{i}=2 \log \frac{p\left(v_{1}, \cdots, v_{n} \mid \theta=k, B_{2}=B_{2}^{i}, D_{2}=D_{2}^{i}\right)}{p\left(v_{1}, v_{2}, \cdots, v_{n} \mid \theta>n\right)}$

where each superscript ' $i$ ' stands for $i$-th detector $(i=1,2, \cdots, N)$ and for each $n$ and each detector,

$$
\ell_{n}^{i}=\max _{k \leq n} L_{n, k}^{i}
$$

Then the decision rule for the detection of an unknown change is

$$
\begin{aligned}
\tau^{i} & =\min \left\{n: \ell_{n}^{i}>h^{i}\right\} \\
\tau & =\min _{i} \tau^{i}
\end{aligned}
$$

where $\tau^{i}$ is a change detection time of each detector, $\tau$ is a real change detection time, and $h^{i}$ is a threshold value for each detector which is found from the probability of false alarm equation. So we can detect this kind of unknown change at time $\tau$ which is the earliest detection time among all the multiple detectors considered in this algorithm. To have a good detection algorithm, the number of change detectors should be sufficiently large and the dynamic range of detectors' change amount should be sufficient to cover the estimated range of changes in system parameters. This means that there exists a trade-off relationship between 'Performance' and 'Complexity' of the algorithm because the computational burden increases dramatifically as the number of change detectors increases.

Detection of the Time and Amount of Change. In some applications, it is insufficient to detect only the change time if the change is unknown, it is also required to detect the amount of change. Fig. 2 is a modified block diagram of Fig. 1 for this purpose. $\overline{\mathrm{CDA}}$ Block is a change detection algorithm which can estimate the amount of an unknown change and the change time. It generates the Activation $\operatorname{Signal}(A S)$ and an Estimated 
Degree of Change $(E D C)$ which is closely related to the real amount of change limited by the resolution of the detectors. When the system change is detected, the system parameter set is updated based on $E D C$ and Parameter Update Block that rules the parameter update law. There is no necessity of System Identification routine. This looks simpler than the adaptive control system explained earlier, but the $\overline{\text { CDA }}$ Block is much more complicated than CDA Block. So, Fig. 2 could be considered another version of block diagram for the total adaptive control system that is appropriate for a system that undergoes an unknown change in parameters. To determine the

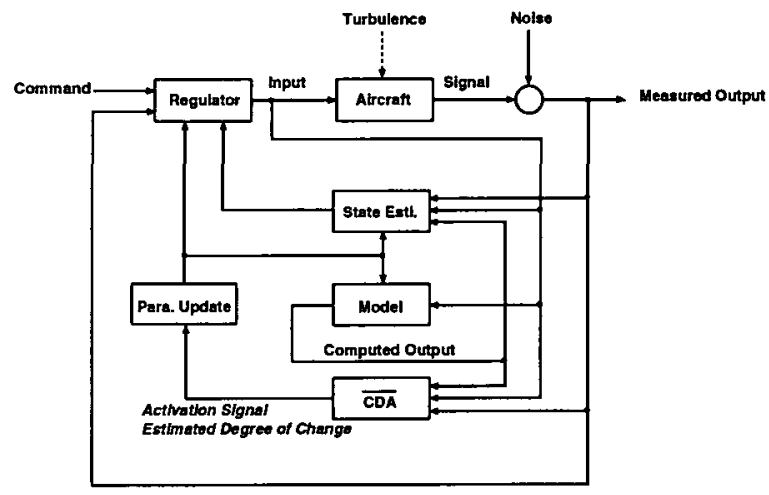

Fig. 2. A Block Diagram of Control System for Aircrafts with $\overline{\mathrm{CDA}}$

amount of change, a 'Decision Mechanism' is required. Based on the $A S$, each of that comes from a known change detector, the Decision Mechanism generates a real one and an $E D C$ at time $n$. The algorithm formulation is the exactly same as before (Eq.(6)-(8)) except the Decision Mechanism, so only the details of Decision Mechanism is explained.

$$
\begin{aligned}
\tau^{i} & =\min \left\{n: \ell_{n}^{i}>h^{i}\right\} \\
\tau & =\min _{i} \tau^{i} \\
\kappa & =\min \left\{i: \tau^{i}\right\}
\end{aligned}
$$

From Eq.(9)-(11), an unknown change could be detected at time $\tau$ whose $E D C$ is equivalent to the $\kappa$-th detector, if the amount of an unknown change is in the range of the amount of multiple detectors. The difficulty of the decision of the exact $E D C$ exists in this decision rule, called D.R. \# 1, because some of change detectors could respond at the same time. That means the necessity of a better decision rule. Since the exact value of $\ell_{n}^{i}$ is rapidly increasing, the following new decision rule (D.R. \# 2) which is the modification of Eq.(11) could be proposed.

$\kappa= \begin{cases}\max \left\{i:\left(\ell_{n}^{i}-h^{i}\right)\right\} & \text { if } \exists \text { more than } 2 \min \tau^{i} \\ \min \left\{i: \tau^{i}\right\} & \text { otherwise }\end{cases}$

There exists the same trade-off between performance and the complexity of the algorithm. Only a finite number of change detectors are used in real simulations.

\section{SIMULATIONS AND RESULTS}

A lateral rigid body dynamics of PA-30, which is a Linear Time-Invariant, is used for simulations (Kim, 1993).

\subsection{Detection of a Known Change}

The mean $(M[\cdot])$ and the variance $(V[\cdot])$ of detection delay $(\tau-\theta)$, with respect to the different change time $(\theta)$, based on 100 runs hereafter, are summarized in Table $1.50 \%$ change is detected earlier than $25 \%$ or $10 \%$ change. From the result of Table 1 , it is shown that different known changes could be detected with some delays.

Table 1 Results for Various Change Times when Change is in Rolling Motion

\begin{tabular}{ccccccc}
\hline$\theta$ & 10 & 20 & 30 & 40 & 50 & 60 \\
\hline$M[\tau-\theta]$ & 7.6 & 0.9 & 7.1 & 4.0 & 11.7 & 10.3 \\
\hline$V[\tau-\theta]$ & 0.4 & 0.3 & 4.5 & 0.4 & 26.6 & 8.2 \\
\hline
\end{tabular}

(a) $10 \%$ Change

\begin{tabular}{ccccccc}
\hline$\theta$ & 10 & 20 & 30 & 40 & 50 & 60 \\
\hline$M[\tau-\theta]$ & 6.2 & 0.1 & 3.1 & 3.0 & 1.6 & 4.6 \\
\hline$V[\tau-\theta]$ & 0.5 & 0.1 & 0.6 & 0.1 & 1.9 & 1.0 \\
\hline
\end{tabular}

(b) $25 \%$ Change

\begin{tabular}{ccccccc}
\hline$\theta$ & 10 & 20 & 30 & 40 & 50 & 60 \\
\hline$M[\tau-\theta]$ & 4.5 & 0.0 & 1.4 & 2.2 & 0.2 & 2.3 \\
\hline$V[\tau-\theta]$ & 0.7 & 0.0 & 0.3 & 0.2 & 0.1 & 0.5 \\
\hline
\end{tabular}

(c) $50 \%$ Change

\subsection{Detection of an Unknown Change: Time}

Simulations with 6 change detectors are done first and then combined to analyze their results as summarized in Table 2. From this Table, the followings are observed:

- For a given detector, large amount of change is detected earlier than small amount of change.

- Rolling motion:

$$
\begin{aligned}
& \text { - } 10 \% \text { Change: } 50 \% \mathrm{D} \geq 25 \% \mathrm{D} \geq 10 \% \mathrm{D} \\
& -25 \% \text { Change: } 10 \% \mathrm{D}, 50 \% \mathrm{D} \geq 25 \% \mathrm{D} \Rightarrow \\
& 10 \% \mathrm{D}, 50 \% \mathrm{D} \simeq 25 \% \mathrm{D} \\
& -50 \% \text { Change: } 10 \% \mathrm{D} \geq 25 \% \mathrm{D} \geq 50 \% \mathrm{D} \\
& \Rightarrow 10 \% \mathrm{D} \simeq 25 \% \mathrm{D} \simeq 50 \% \mathrm{D}
\end{aligned}
$$


Table 2 Summarized Simulation Results for $\theta=30$ where R.D., Y.D., R.C., Y.C., and N.D. stand for Rolling Detector, Yawing Detector, Rolling Change, Yawing Change, and No Detection, respectively

\begin{tabular}{lcccccc}
\hline & \multicolumn{2}{c}{$10 \%$} & \multicolumn{2}{c}{$25 \%$} & \multicolumn{2}{c}{$50 \%$} \\
\cline { 2 - 7 } & R.D. & Y.D. & R.D. & Y.D. & R.D. & Y.D. \\
\hline 10\% R.C. & 7.05 & 16.2 & 9.02 & 15.8 & 12.3 & 15.7 \\
\hline $10 \%$ Y.C. & N.D. & 16.6 & N.D. & 16.6 & N.D. & 16.5 \\
\hline 20\% R.C. & 3.88 & 15.6 & 3.84 & 15.6 & 4.31 & 15.0 \\
\hline 20\% Y.C. & N.D. & 16.6 & N.D. & 16.6 & N.D. & 16.5 \\
\hline 25\% R.C. & 3.23 & 15.2 & 3.11 & 15.0 & 3.29 & 14.9 \\
\hline 25\% Y.C. & N.D. & 16.6 & N.D. & 16.6 & N.D. & 16.5 \\
\hline 30\% R.C. & 2.73 & 15.0 & 2.55 & 14.9 & 2.73 & 15.8 \\
\hline 30\% Y.C. & N.D. & 16.6 & N.D. & 16.6 & N.D. & 16.5 \\
\hline 40\% R.C. & 2.18 & 14.8 & 1.90 & 14.7 & 1.94 & 14.8 \\
\hline $40 \%$ Y.C. & N.D. & 16.6 & N.D. & 16.6 & N.D. & 16.5 \\
\hline 50\% R.C. & 1.81 & 14.7 & 1.57 & 14.7 & 1.43 & 14.7 \\
\hline 50\% Y.C. & N.D. & 16.6 & N.D. & 16.6 & N.D. & 16.5 \\
\hline 60\% R.C. & 1.55 & 14.5 & 1.25 & 14.6 & 1.11 & 14.6 \\
\hline 60\% Y.C. & N.D. & 16.6 & N.D. & 16.5 & N.D. & 16.5 \\
\hline
\end{tabular}

where ' $D$ ' denotes a detector and the detection delay is quite reduced when $10 \%$ detector is used to detect $10 \%$ change but there is no big difference in detection delay of 3 change detectors to detect $25 \%$ or $50 \%$ change.

- Yawing motion: The results are the same as rolling motion, but detection delay is large and the difference of delay with respect to each change detector is small since the portion of yawing motion in PA-30 is small.

From the above observations and the trade-off between the number of change detectors and the computational burden, it could be concluded that $10 \%$ change detector is sufficient when the detection of change larger than $10 \%$ is concerned.

\subsection{Detection of an Unknown Change: Time} and Amount

Simulation results, with D.R. \# 2, for $\theta=40$ are summarized in Table 3. From this table, it could be shown that the unknown changes at $\theta=40$ are fairly well detected except $30 \%$ and $40 \%$ changes, which might be fixed if the better decision rule is applied. Simulation results for $\theta=10,20$, and 30 have the same characteristics. Therefore, it could be concluded that the given unknown changes are detected in statistical sense, though there exists a difficulty in deciding the exact $E D C$ in some situations which might be improved by applying the better decision rule.

\subsection{Change Detection via Whiteness Test}

To examine the validity of MT-CDA, simulations
Table 3 Simulation Results for $\theta=40$

\begin{tabular}{cccc}
\hline & Detection Number & $M[\tau-\theta]$ & $V[\tau-\theta]$ \\
\hline $10 \%$ D & 69 & 4.12 & 0.31 \\
\hline $20 \%$ D & 29 & 3.55 & 0.39 \\
\hline $30 \%$ D & 0 & & \\
\hline $40 \%$ D & 1 & 4.0 & 0.0 \\
\hline $50 \%$ D & 1 & 2.0 & 0.0 \\
\hline
\end{tabular}

(a) $10 \%$ change

\begin{tabular}{cccc}
\hline & Detection Number & $M[\tau-\theta]$ & $V[\tau-\theta]$ \\
\hline $10 \% \mathrm{D}$ & 0 & & \\
\hline $20 \% \mathrm{D}$ & 87 & 3.15 & 0.15 \\
\hline $30 \% \mathrm{D}$ & 3 & 3.33 & 0.22 \\
\hline $40 \% \mathrm{D}$ & 3 & 3.0 & 0.0 \\
\hline $50 \% \mathrm{D}$ & 7 & 2.29 & 0.49 \\
\hline
\end{tabular}

(b) $20 \%$ change

\begin{tabular}{lccc}
\hline & Detection Number & $M[\tau-\theta]$ & $V[\tau-\theta]$ \\
\hline $10 \% \mathrm{D}$ & 0 & & \\
\hline $20 \% \mathrm{D}$ & 18 & 2.89 & 0.10 \\
\hline $30 \% \mathrm{D}$ & 27 & 3.07 & 0.07 \\
\hline $40 \% \mathrm{D}$ & 33 & 2.97 & 0.03 \\
\hline $50 \% \mathrm{D}$ & 22 & 2.23 & 0.18
\end{tabular}

(c) $30 \%$ change

\begin{tabular}{lccc}
\hline & Detection Number & $M[\tau-\theta]$ & $V[\tau-\theta]$ \\
\hline $10 \% \mathrm{D}$ & 0 & & \\
\hline $20 \% \mathrm{D}$ & 4 & 2.5 & 0.25 \\
\hline $30 \% \mathrm{D}$ & 3 & 3.0 & 0.0 \\
\hline $40 \% \mathrm{D}$ & 23 & 3.0 & 0.0 \\
\hline $50 \% \mathrm{D}$ & 70 & 2.36 & 0.23 \\
\hline
\end{tabular}

(d) $40 \%$ change

\begin{tabular}{lccc}
\hline & Detection Number & $M[\tau-\theta]$ & $V[\tau-\theta]$ \\
\hline $10 \% \mathrm{D}$ & 0 & & \\
\hline $20 \% \mathrm{D}$ & 1 & 2.0 & 0.0 \\
\hline $30 \% \mathrm{D}$ & 0 & & \\
\hline $40 \% \mathrm{D}$ & 2 & 2.0 & 0.0 \\
\hline $50 \% \mathrm{D}$ & 97 & 2.23 & 0.18 \\
\hline
\end{tabular}

(e) $50 \%$ change

of Whiteness Test are done and results are compared in this section. Only the detection problem of a known change is considered here.

Table 4 shows the detection delay of each algorithm for various change time $(\theta)$. For every tests, the detection delay of MT-CDA is smaller than that of Whiteness Test. Therefore, the simulation results show that MT-CDA is superior to Whiteness Test in the sense of detection delay comparison.

\section{CONCLUSIONS}

To design a reconfigurable control system, a simple model with a single change in parameters of control gain matrices at random time whose a priori distribution is known has been considered. Changes in these matrices mean the failure in the actuators, so possible damages in the control surfaces and/or one of the engine failure could be 
Table 4 Simulation Results for Various Change Times when 25\% Change is in Rolling Motion

\begin{tabular}{cccccc}
\hline$\theta$ & 10 & 20 & 30 & 40 & 50 \\
\hline$M[\tau-\theta]$ & 6.2 & 0.1 & 3.1 & 3.0 & 1.6 \\
\hline$V[\tau-\theta]$ & 0.5 & 0.1 & 0.6 & 0.1 & 1.9 \\
\hline
\end{tabular}

(a) Minimal Time-Change Detection Algorithm

\begin{tabular}{cccccc}
\hline$\theta$ & 10 & 20 & 30 & 40 & 50 \\
\hline$M[\tau-\theta]$ & 11.7 & 4.0 & 17.9 & 7.9 & 37.4 \\
\hline$V[\tau-\theta]$ & 0.2 & 0.0 & 0.1 & 0.1 & 4.8 \\
\hline
\end{tabular}

(b) Whiteness Test

analyzed with this model. Therefore, considering changes in the control gain matrices is the proper starting point before we consider all kinds of changes. MT-CDA, based on Likelihood Ratio Test, for this simple model has been developed whether a change is known or unknown and it has been shown to be optimal for a fixed false alarm probability.

Simulation results for lateral motions show that a known change can be detected with some delays which should be minimal for the given condition as theory indicates. From simulation results for the detection of change time, it could be concluded that $10 \%$ change detector, which is the smallest one, is sufficient and it is robust in the sense that it could detect any change( $>$ $10 \%$ ) with sub-minimal detection delay. And the unknown change could be detected, in statistical sense, though there exists a difficulty to decide the exact $E D C$ in some situations. The better decision rule needs to be developed to solve this difficulty. The algorithm with multiple change detectors, if implemented in full, will yield the best performance for the widest class of change. But full implementation is another problem. Simulations have been done throughout the various situations and the results are meaningful (Kim, 1993). Detection via Whiteness Test has been also formulated for algorithm comparison in the sense of detection delay. MT-CDA is superior to the Whiteness Test in this sense.

In this paper, MT-CDA has been developed and demonstrated by simulations when a change is in control gain matrices. With this MT-CDA, a reconfigurable control system which is a highly adaptive system shown in Fig. 1 or Fig. 2 could be built and work throughout a wide range of conditions.

\section{REFERENCES}

Balakrishnan, A.V. (1972). Identification and Adaptive Control: An application to Flight Control Systems, Journal of Optimization Theory and Applications.

Basseville, M. (1988). Detecting Changes in Signals and Systems - A Survey, Automatica, Vol. 24, No. 3, pp. 309-326.

Basseville, M. and Benveniste, A. (1980). Detection of Abrupt Changes in Signals and Dynamical Systems, Lecture Notes in Control and Information Sciences 77, SpringerVerlag.

Basseville, M. and Nikiforov I.V. (1993). Detection of Abrupt Changes: Theory and Application, Englewood Cliffs, Prentice Hall, New Jersey.

Chow, Y.S., Robbins H., and Siegmund, D. (1971). Great Expectations: The Theory of Optimal Stopping, Houghton Mifflin, Boston.

Iliff, K.W. (1973). Identification and Stochastic Control with Application to Flight Control in Turbulence, Ph.D. Dissertation, U.C.L.A.

Kim, S. (1993). A Development of Minimal Time-Change Detection Algorithm (MTCDA) in System Parameters and Its Application to Flight Systems, Ph.D. Dissertation, U.C.L.A.

Kim, S. (1994). The Minimal Time Detection Algorithm, IEEE Aerospace Applications Conference, pp. 103-115.

Lorden, G. (1971). Procedures for Reacting to a Change in Distribution, The Annals of Mathematical Statistics, Vol. 42, No. 6, pp. 18971908.

Maine, R.E. and Iliff, K.W. (1986). Application of Parameter Estimation to Aircraft Stability and Control: The Output-Error Approach, NASA Reference Publication 1168.

Page, E.S. (1954). Continuous Inspection Schemes, Biometrica, Vol. 41, pp. 100-115.

Shiryayev, A.N. (1978). Optimal Stopping Rules, Springer-Verlag, New York.

Wald, A. (1947). Sequential Analysis, Wiley, New York.

Wilsky, A.S (1976). A Survey of Design Methods for Failure Detection in Dynamic Systems, Automatica, Vol. 12, pp. 601-661. 